\title{
Real-Time Traffic Control System using Fuzzy Logic based Edge Detector for Images
}

\author{
Sreemana Datta \\ School of Education Technology \\ Jadavpur University \\ Kolkata, India
}

\author{
Joydeep Mukherjee \\ Assistant Professor \\ School of Education Technology \\ Jadavpur University \\ Kolkata, India
}

\begin{abstract}
With the rising traffic congestion, an efficient method to control traffic and ensuring proper signalling has become very important. A number of methods are used worldwide to control and signal road traffic. We propose a method based on image processing to determine the density of vehicles on a particular lane of a road and control the signal accordingly. For each road crossing, screenshots will be taken from live feeds to determine the traffic density of a road and then based on an efficient fuzzy logic based edge detector; a comparative analysis of traffic density will be performed. Traffic signalling will be done so as to ensure that the busiest road gets the green signal at the earliest and for the longest duration. The second busiest road will get the green signal next and for a lesser duration. Signalling of other roads will be done accordingly. We have utilised a fuzzy rule based edge detection algorithm which is accurate and efficient.
\end{abstract}

\section{General Terms}

Image Processing, Fuzzy Logic.

\section{Keywords}

Edge Detection, Sliding Window Based Detector, Fuzzy logic, Traffic Control, Fuzzy Sets.

\section{INTRODUCTION}

Multiple techniques have been implemented worldwide for management and systematic signalling of road traffic and designing of Intelligent Traffic Systems (ITS). It has been observed that traditional system of traffic control involves manual control which leads to employment of manpower and also suffers from human errors. Other semi-automatic traffic controlling and signalling techniques such as the ones based on statistical data and fixed time slot based round robin scheduling are not capable to adapt dynamically to changing traffic densities and hence often the less busy lanes enjoy greater duration of green signal whereas busy lanes has greater waiting time for vehicles.

Few other device based techniques used to implement intelligent traffic systems include systems using PLCs, PICs, Radio Frequency ID tags, Global Positioning System, Infrared sensors, Electromagnetic sensors and Inductive Loops. Almost all of these techniques have some additional hardware requirements to be installed on the vehicle which makes them infeasible for large scale implementation. Few of these techniques involve use of sophisticated and often costly instruments such as GPS sensors which hinders wide scale application. Infrared sensors, inductive loops and electromagnet based techniques have been observed to produce degraded performance under harsh weather conditions such as hailstorm, rainfall and snow. We propose a simple yet efficient technique based to combined use of image processing and fuzzy logic techniques to achieve at the desired goal of efficient traffic signalling and control.

\section{TRADITIONAL METHODS FOR EDGE DETECTION}

Edge Detection is a useful tool in low-level digital image processing and analysis of images in the field of computer vision and pattern recognition. Edges in an image carry essential information of an object of interest as they differentiate between dissimilar regions. Use of specific linear time-invariant (LTI) filters is one of the most common techniques used for edge detection. It also involves minimum computational complexity. In first-order filters, an edge is interpreted as a sudden variation in grayscale level between two neighbouring pixels. The target here is determining the points in the image in which the first derivative of the grayscale level as a function of position has high value or magnitude. By thresholding the resultant output image, edges are detected.

Sobel and Prewitt operator based edge detectors [1], [2] are based on the theory of spatial derivative filtering, where local gradient operators are used for detecting edges. A limitation of this method is that edge detection is possible for certain orientations only by this technique. Derivative filters are seen to degrade in quality when the edges become blurred and noisy. Marr and Hildreth [3] algorithm detects edges at the Laplacian zero-crossings of an image. Canny edge detector [4] attempted to tackle the problem of noise from gradient operators, where convolving of an image is performed with the first-order derivatives of Gaussian filter for smoothing in the direction of local gradient and then following it by edge detection using thresholding technique.

Most edge detector work by applying local first order derivative and then second order derivative operator and then following it by using some algorithm for noise reduction. SUSAN edge detector [5] is an example of non-linear technique for edge detection. Hou and Kuo [6] proposed a technique using simple arithmetic and logical operation to find the edges in an image. Jiang and Bunke[7] used a scan line approximation method for edge detection showing superior segmentation quality and lesser computational complexity than many region based algorithms . Caragea's method [8] calculates the difference between central pixel and surrounding pixel and utilizes the highest value among these for detecting the edges. Genming [9] used a 5x 5 window for 
efficient noise reduction while not increasing the width of the edges which always used to happen in case of taking $5 \times 5$ window. Since this detector uses a self-adjusting threshold, therefore it is also capable of edge detection in the area having variable grayscale background.

\section{FUZZY LOGIC AND ITS USES}

Fuzzy logic has emerged as an efficient and powerful tool to decision making [10], [11], [12]. It was in 1965 when Zadeh put forward the concept of fuzzy logic and it gained popularity in the image processing field soon. Many techniques have been proposed by various researchers for fuzzy logic based edge detection [13], [14], [15]. Zhao[16], proposed an edge detection algorithm by dividing an image into 3-fuzzy partitions or regions and then computing the maximum entropy to find the best edge. His work also included discovery of the necessary conditions for maximizing the entropy function. Based on these conditions, the three-level thresholding is obtained. Bloch [17] proposed a morphological operation based fuzzy set for edge detection in images. Russo and Ramponi [18] used fuzzy rule-based operators built on the classic IF-THEN-ELSE rule-based architecture for edge detection. A drawback of this algorithm is that the global information has been neglected. In most of these techniques[19],[20], the neighbouring pixels around a particular center pixel are assumed within some classes and then by using some appropriate membership function, a fuzzy inference system is designed which can extract the edges from the image to suffice the purpose. Mansoori [21], grouped all the adjacent pixels around a certain pixel under consideration in 6 different sets. Then the membership value for each group was determined by applying some bell shape function. Then by using these membership values and the fuzzy rule, existence of the edge pixel was determined.

\section{PROPOSED ALGORITHM}

In the proposed algorithm based on fuzzy logic rules, a scanning mask of $2 \times 2$ pixels window is used. This is done to reduce complexity. A rule based fuzzy inference system has been designed using Java under Eclipse IDE to detect the edge. The rule-base consists of 16 rules and has been designed so as to detect a particular pixel under consideration within a $2 \times 2$ window as White, Edge or Black pixel. Based on this computation, an output pixel is generated which is further used for edge detection of an image arising out of a live feed of traffic at a particular timestamp. Having done with detecting edges of the image using the fuzzy logic based technique, we perform an image matching of the resultant image with that of the edge detected image of that particular road when it is empty, i.e. when there is no vehicle in the road. Greater the percentage match, lesser populated is the road. Signalling of traffic is hence done based on a customizable protocol.

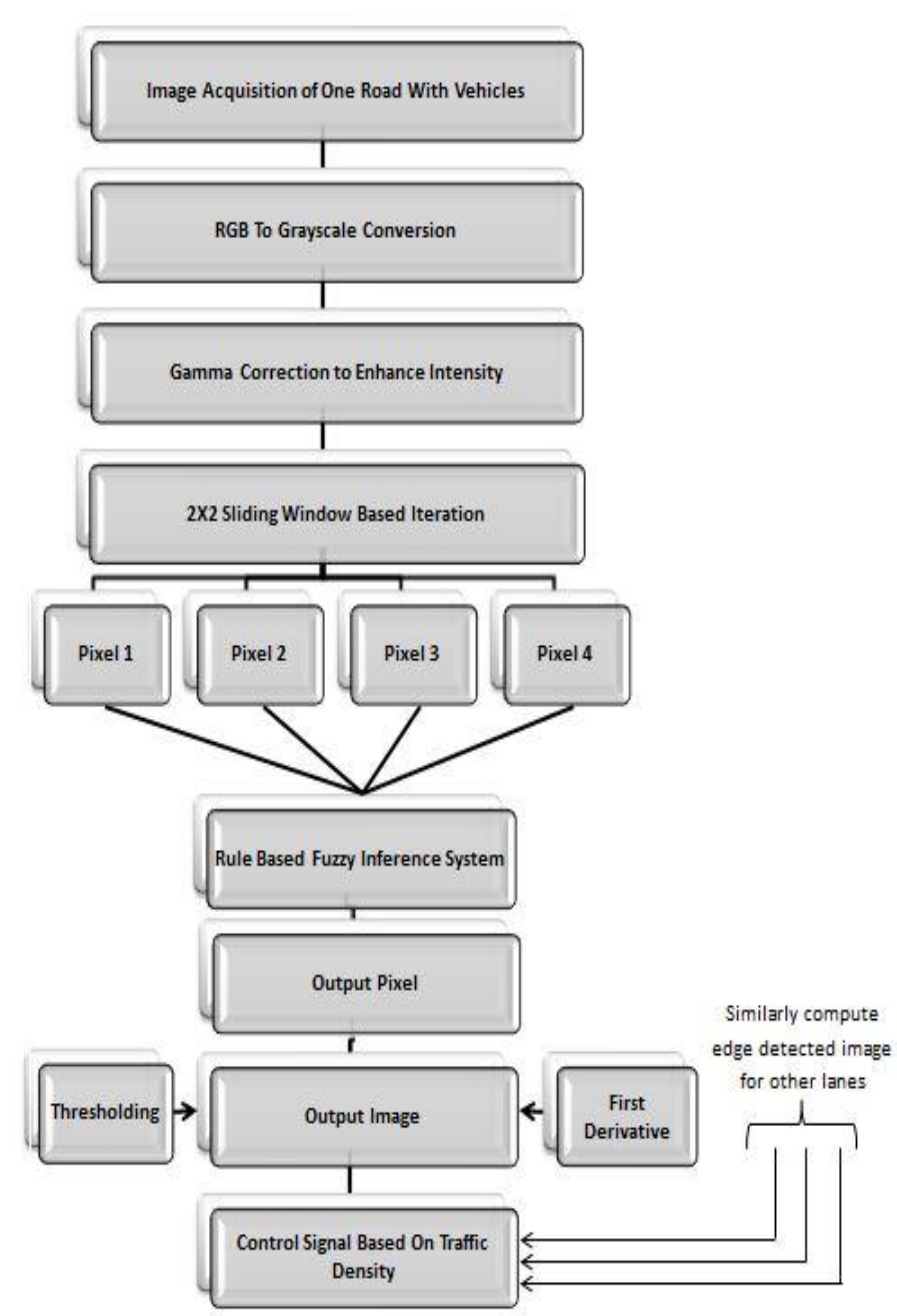

Fig. 1: Flowchart of Proposed Algorithm

The proposed system aims to achieve the following:

i. Distinguish between the presence and absence of vehicles on roads.

ii. Calculate the density or congestion level of a particular road.

iii. Determine the order of traffic light signalling based on the calculated traffic density.

iv. To ensure that the most busy lane gets the green signal for the longest time duration.

\section{EXPERIMENTAL METHODS}

The following methods were developed and implemented during the experiment phase:

\subsection{Image Acquisition}

Real time video feed from the camera modules mounted at roadside traffic lights and crossings are acquired and converted into frames for image processing based on their timestamps. Then we apply image processing algorithms on the static frames repetitively after certain intervals and decision making on whether vehicle is present or not is done. 


\subsection{RGB to Grayscale Conversion}

RGB to Grayscale conversion is done to reduce the cost of computation while not having to compromise on the efficiency. The lookup table of a RGB image consists of 24 bits $(8$ bits each for R,G and B since each may have values ranging from 0-255) whereas lookup table of grayscale image consists of only 8 bits (values from 0-255). It has been observed that edge detection algorithms produce similar results for both RGB and grayscale images; hence, we perform this step to reduce computational complexity.

\subsection{Image Enhancement}

This is done to bring the image in contrast to the background so that a proper thresholding level may be decided upon while binary conversion is being carried out and the result hence obtained is more suitable than the original image for the specific purpose.It is often seen that the enhanced image gives better results in edge detection when compared to the original image. Image enhancement simply means, transforming an image $f$ into image $g$ using $\mathrm{T}$. (Where $\mathrm{T}$ is the transformation) The values of pixels in images $f$ and $g$ are denoted by $r$ and $s$, respectively. As said, the pixel values $r$ and $s$ are related by the expression,

$$
\mathrm{s}=\mathrm{T}(\mathrm{r})
$$

Where $\mathrm{T}$ is a transformation that maps a pixel value $\mathrm{r}$ into a pixel values. As Power Law transformation works well for general contrast manipulation, we used power law transformation or gamma correction as our image enhancement technique.

Power Law Transformations(Gamma Correction) :

$$
\left[\mathrm{s}=\mathrm{cr}^{\wedge} \gamma\right]
$$

For daytime, the value of $\gamma$ was taken as 0.5 (less than 1.0) in order to darken the image and increase contrast so that cars are not missed or washed out due to reflection or excessive illumination. For night time, the value of $\gamma$ was taken as 1.5 (more than 1.0) in order to enhance light in the image and ensure that cars are not missed out in the darkness or due to less ambient light.

\section{$5.42 \times 2$ Sliding Window Based Iteration}

The Fuzzy inference system for the proposed algorithm has four input parameters and one output parameter. In this algorithm, $2 \times 2$ sliding window mask is used for scanning the pixels and the four- pixel values obtained by this window mask is used as four inputs. Then window begins to scan from the top left position of the image and scans from left to right. It goes all the way to the bottom right of the image and ends the scanning. This way, at a time four pixels are scanned in one timestamp and we gain in computational complexity of pixel by pixel iteration.

A $2 \times 2$ mask used in the algorithm is shown in Fig. 2. For example, here since $\mathrm{PA}$ is black and $\mathrm{PB}, \mathrm{PC}, \mathrm{PD}$ are white therefore as per the rule shown in Table II the output pixel Pout is marked as white.

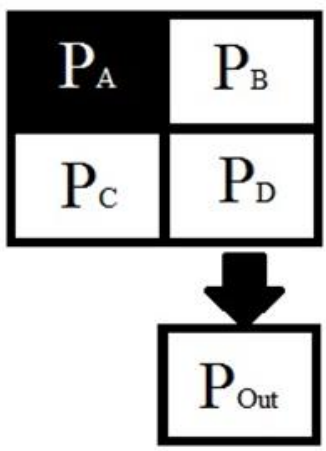

Fig. 2: 2×2 Sliding Window Mask

\subsection{Rule Based Fuzzy Inference System}

In order to perform edge detection in the image, a rule based fuzzy inference system has been developed which takes different pixel values as input parameters, then fuzzifies these parameters, i.e. transforms it into the fuzzy plane and then utilizes some pre-set rules to mark the considered pixel as Edge pixel, Black pixel or White pixel. We have used the Mandani method to perform the defuzzification step and the resultant output of the system is computed as the centroid of the chosen resultant membership functions.

5.5.1 Choosing A Membership Function: The choice of a membership function depends on the type of application or problem. In our proposed algorithm, Triangular Membership function has been used for both input and output. The standard Triangular membership function may be defined as

$$
T F(x ; a, b, c)=\left\{\begin{array}{ll}
0 & x \leq a \text { or } x>c \\
\frac{(x-a)}{(b-a)} & a<x<b \\
\frac{(c-x)}{(c-b)} & b<x \leq c
\end{array}\right\}
$$

The shape of the chosen membership function is shown in Fig 3.

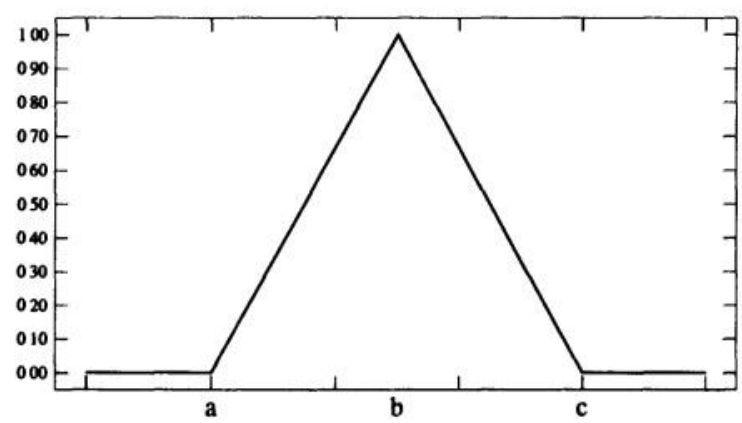

Fig. 3: Shape of Triangular Function

The parameters of this function are denoted as a,b and c, where $b$ is a peak point, magnitude of left width is (b-a) and magnitude of right width is (c-b).

\subsubsection{Segregation of Input and Output into Fuzzy}

Sets: All the input pixels are divided in to two fuzzy sets i.e. Black \& White and all the output pixels are divided into three fuzzy sets i.e. Black, Edge and White. The Membership functions associated with the input fuzzy sets and the output fuzzy set is shown in figure 4 and figure 5 . 


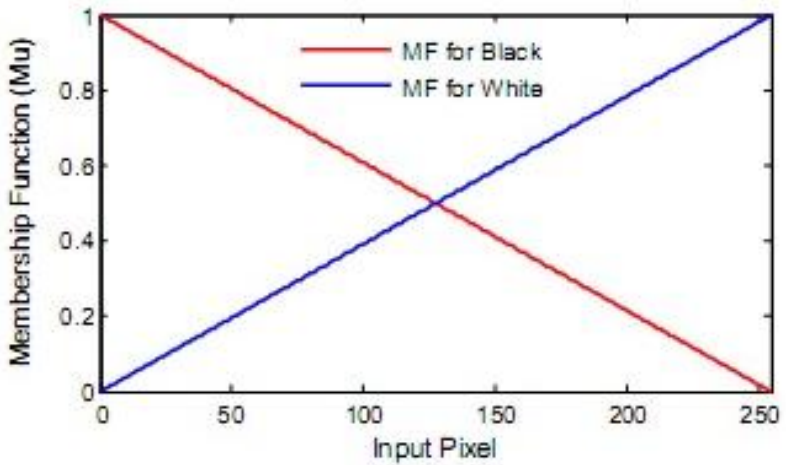

Fig. 4: Fuzzy Set for Input Pixels

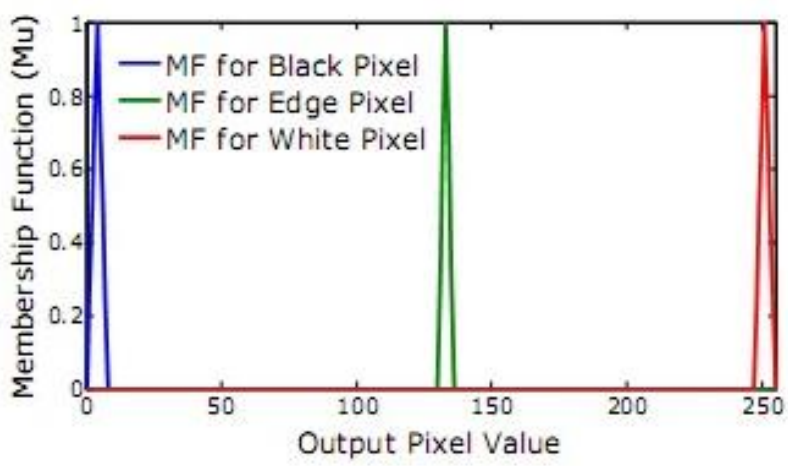

Fig. 5: Fuzzy Set for Output Pixels

The parameters present in input pixels fuzzy set and output pixels fuzzy set have been summarized in Table I.

Table 1: Summary of Parameters In Fuzzy Set

\begin{tabular}{|c|c|c|c|c|}
\hline $\begin{array}{c}\text { Fuzzy } \\
\text { Input }\end{array}$ & $\begin{array}{c}\text { Pixel } \\
\text { Variable }\end{array}$ & Range & Parameter & $\begin{array}{l}\text { Membership } \\
\text { Function }\end{array}$ \\
\hline \multirow{2}{*}{$\mathrm{PA}_{\mathrm{A}}$} & White & \multirow{2}{*}{$\begin{array}{c}{[0} \\
255]\end{array}$} & {$\left[\begin{array}{llll}0 & 255 & 255\end{array}\right]$} & Triangular \\
\hline & Black & & {$\left[\begin{array}{lll}0 & 0 & 255\end{array}\right]$} & Triangular \\
\hline \multirow{2}{*}{ Рв } & White & \multirow{2}{*}{$\begin{array}{c}{[0} \\
255]\end{array}$} & {$\left[\begin{array}{llll}0 & 255 & 255\end{array}\right]$} & Triangular \\
\hline & Black & & {$\left[\begin{array}{lll}0 & 0 & 255\end{array}\right]$} & Triangular \\
\hline \multirow{2}{*}{$\mathrm{PC}_{\mathrm{C}}$} & White & \multirow{2}{*}{$\begin{array}{c}{[0} \\
255]\end{array}$} & {$\left[\begin{array}{llll}0 & 255 & 255\end{array}\right]$} & Triangular \\
\hline & Black & & {$\left[\begin{array}{lll}0 & 0 & 255\end{array}\right]$} & Triangular \\
\hline \multirow{2}{*}{$\mathrm{PD}$} & White & \multirow{2}{*}{$\begin{array}{c}{[0} \\
255]\end{array}$} & {$\left[\begin{array}{llll}0 & 255 & 255\end{array}\right]$} & Triangular \\
\hline & Black & & {$\left[\begin{array}{lll}0 & 0 & 255\end{array}\right]$} & Triangular \\
\hline $\begin{array}{l}\text { Fuzzy } \\
\text { Output }\end{array}$ & $\begin{array}{l}\text { Pixel } \\
\text { Label }\end{array}$ & & Range & $\begin{array}{l}\text { Membership } \\
\text { Function }\end{array}$ \\
\hline \multirow{3}{*}{ Pout } & Black & \multirow{3}{*}{$\begin{array}{c}{[0} \\
255]\end{array}$} & [ [ $\left.\begin{array}{lll}0 & 4 & 8\end{array}\right]$ & Triangular \\
\hline & Edge & & $\begin{array}{c}{\left[\begin{array}{ll}130 & 133 \\
136\end{array}\right]} \\
\end{array}$ & Triangular \\
\hline & White & & $\begin{array}{c}247251 \\
255]\end{array}$ & Triangular \\
\hline
\end{tabular}

5.5.3 Applying the Rule Based Fuzzy Algorithm: Having achieved the output of the $2 \times 2$ sliding window mask in the form of four pixels, these four pixels goes through different fuzzy conditions as described in Fig. 6 and change the value of $\mathrm{PD}$.

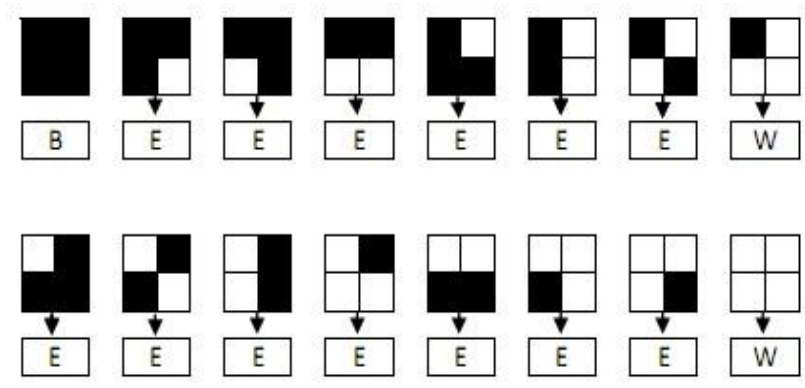

Fig. 5: The different Fuzzy Conditions

The rule based Fuzzy Inference System matches the condition of each pixel with that of the Rule base given in Table II and marks the pixel under consideration as an edge pixel, white pixel or black pixel.

Table 2: The Fuzzy Rule Base

\begin{tabular}{|c|c|c|c|c|}
\hline \multicolumn{4}{|c|}{ Fuzzy Input } & $\begin{array}{l}\text { Fuzzy } \\
\text { Output }\end{array}$ \\
\hline $\mathrm{P}_{\mathrm{A}}$ & Рв & $P_{C}$ & $\mathrm{PD}$ & Pout \\
\hline B & B & B & B & B \\
\hline B & B & $\mathrm{B}$ & $\mathrm{W}$ & $E$ \\
\hline $\mathrm{B}$ & $\mathrm{B}$ & $\mathrm{W}$ & $\mathrm{B}$ & $\mathrm{E}$ \\
\hline $\mathrm{B}$ & $\mathrm{B}$ & $\mathrm{W}$ & $\mathrm{W}$ & $\mathrm{E}$ \\
\hline $\mathrm{B}$ & $\mathrm{W}$ & $\mathrm{B}$ & $\mathrm{B}$ & $\mathrm{E}$ \\
\hline B & $\mathrm{W}$ & B & $\mathrm{W}$ & $\mathrm{E}$ \\
\hline $\mathrm{B}$ & $\mathrm{W}$ & $\mathrm{W}$ & $\mathrm{B}$ & $\mathrm{E}$ \\
\hline B & $\mathrm{W}$ & $\mathrm{W}$ & $\mathrm{W}$ & $\mathrm{W}$ \\
\hline $\mathrm{W}$ & $\mathrm{B}$ & B & $\mathrm{B}$ & $E$ \\
\hline $\mathrm{W}$ & $\mathrm{B}$ & B & $\mathrm{W}$ & $\mathrm{E}$ \\
\hline W & B & $\mathrm{W}$ & B & $\mathrm{E}$ \\
\hline $\mathrm{W}$ & $\mathrm{B}$ & $\mathrm{W}$ & $\mathrm{W}$ & $\mathrm{E}$ \\
\hline $\mathrm{W}$ & W & B & B & $E$ \\
\hline $\mathrm{W}$ & $\mathrm{W}$ & $\mathrm{B}$ & $\mathrm{W}$ & $\mathrm{E}$ \\
\hline $\mathrm{W}$ & $\mathrm{W}$ & $\bar{W}$ & B & $\mathrm{E}$ \\
\hline $\mathrm{W}$ & $\mathrm{W}$ & $\mathrm{W}$ & $\mathrm{W}$ & $\mathrm{W}$ \\
\hline
\end{tabular}

Steps of the algorithm are as follows:

1) Input the $\mathrm{PA}, \mathrm{PB}, \mathrm{PC}, \mathrm{PD}_{\mathrm{D}}$ pixels from the scanned window to Fuzzy Information System and fuzzify it into different degrees of Black and White.

2) Apply the Fuzzy t-norms operator (MIN) in order to calculate the firing strength.

3) Apply the fuzzy rules for each input parameter.

4) Apply the Fuzzy s-norms operator (MAX) to get the aggregate resultant output.

5) Apply Mandani method to perform Defuzzification using centroid method.

6) Obtain the Pout pixel which may be categorized under White pixel, Black pixel or Edge pixel category

7) Compute the first derivative and apply thresholding to obtain the edge of the image. 


\subsection{Controlling Signal Based on Traffic Density}

Having detected the edges of the real-time image of the road with vehicles, we compare the same with that of the edge detected image of the same road when it is devoid of any vehicles. Greater the percentage match found, it was understood that the road was emptier and likewise, applying the same algorithm on other roads coming to the crossing on which the camera was installed, the roads were ordered in descending order of their traffic densities. Then traffic light signalling was performed. This two-step algorithm is explained as follows.

\subsubsection{Window based image matching with adaptive} updating of background of the image: Edge detected image of an empty road was stored as reference. We went on changing the background image adaptively. This means, at any instant, the background has a contribution of the $\mathrm{x}$ times the nth frame and (1-x) times previous (say ' $\mathrm{n}-\mathrm{1}^{\prime}$ ) frames, where $\mathrm{x}$ is a constant between 0 and 1 and is defined as the contribution factor. We proceeded with a sequence of 10 frames per cycle and hence chose the value of $x$ to be 0.9 . At a particular timestamp, the adaptively updated background ensures greater accuracy in cases where there are fluctuations in traffic density. Then, a window based image comparison is done on each of these frames to compare them with the background image for that instant. Window based image matching refers to sub dividing the entire image into smaller windows and then iterating over the windows to compute the percentage of match. Rather than iterating pixel by pixel, this method provides better time complexity though the results are received at an acceptable level of approximation. In this technique, the larger image is subdivided into multiple pixel windows of the dimensions of the smaller image. The idea is that, if two images differ, each of these pixel windows will differ and vice versa. Likewise, the decision making is performed. Comparisons of the edge detected image of the empty road with that of image frames for each lane resulted in four distinct values of percentage match corresponding to each road with respect to the background image.

\subsubsection{Traffic Signaling Based On the Percentage}

Match Computed : After the percentage match of each road was computed, it was observed that higher the percentage match for each lane as a result of image matching with the empty lane, less densely populated is the lane. A densely populated road was seen to show very less percentage match whereas a sparsely populated road showed high value of percentage match. Hence, traffic signaling protocol was finalized as:

i) Match Of 10 To 50\% - Green Light On For 60 Seconds

ii) Match Of 50 To $70 \%$ - Green Light On For 30 Seconds

iii) Match Of 70 To $90 \%$ - Green Light On For 20 Seconds

iv) Match Of 90 To $100 \%$ - Red Light On For 60 Seconds

This protocol may however be modified as per practical requirements.

\section{RESULTS}

We did coding of the algorithm in Java. We ran our code in Eclipse IDE (Kepler) in a PC with Intel Core i3 processor and 4GB of RAM. Edge detection results were compared with Canny Edge Detector (deemed as the most efficient edge detector for a wide range of applications). It was observed that though results produced by Canny edge detector were slightly better, it took 2.5 to 3 times more time than the detector based of Fuzzy Inference System. For our application in traffic signalling, the Fuzzy logic based edge detector rendered satisfactory results.

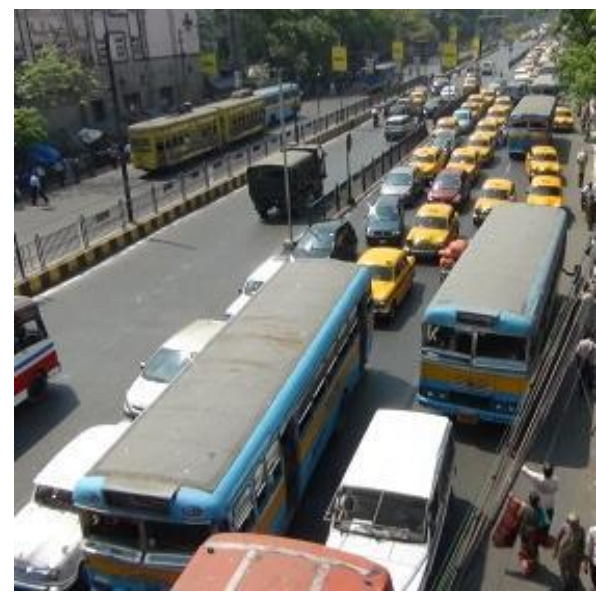

Fig. 6: Original Image

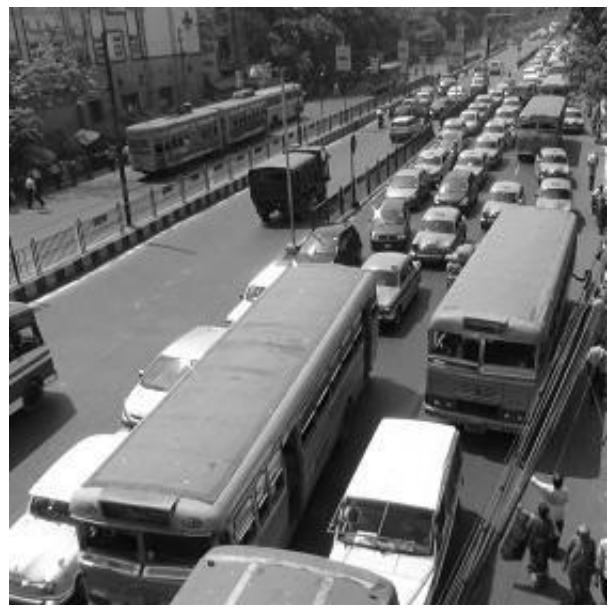

Fig. 7: After RGB to Grayscale Conversion

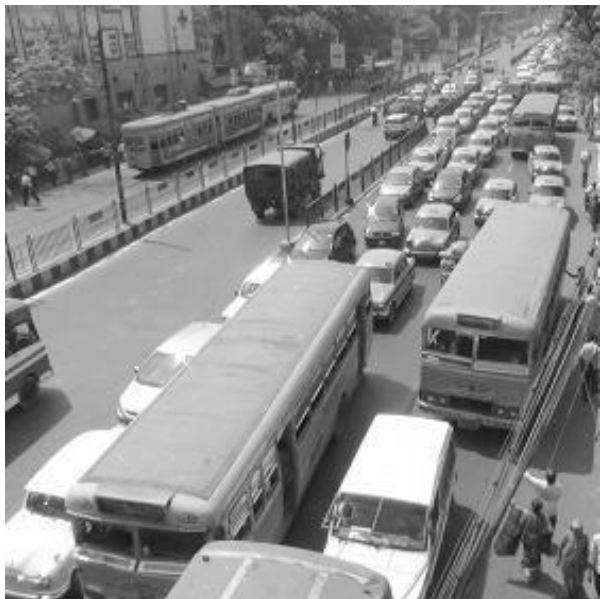

Fig. 8: After Gamma Correction 


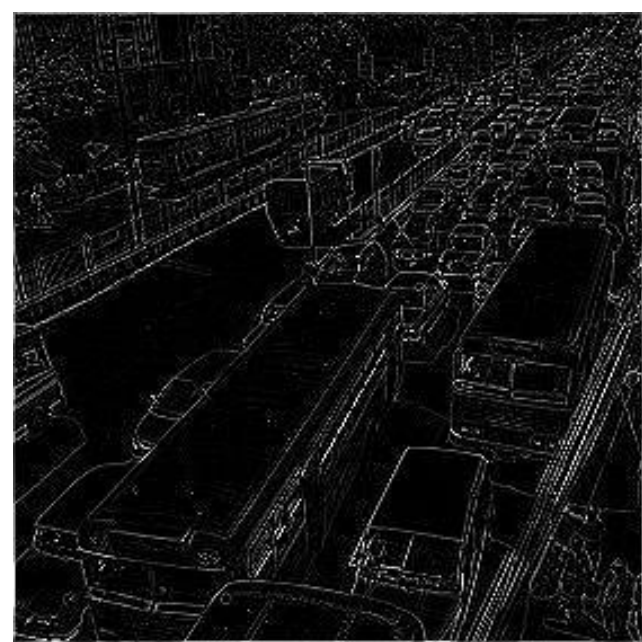

Fig. 9: Fuzzy Logic Based Edge Detection

(Took 609 milliseconds in this case)

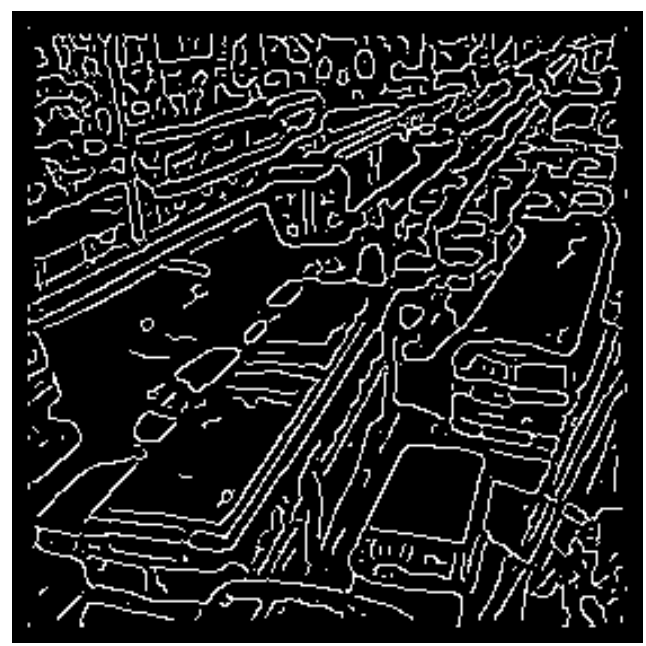

Fig. 10: Canny Edge Detection

(Took 1591 milliseconds in this case)

\section{CONCLUSION}

It was observed that the window size and the accuracy of computation are inversely proportional. Pixel by pixel comparison can be considered as a window based comparison where window size is $1 \mathrm{x} 1$. It provides the best result in terms of accuracy but is very time consuming. It is also to be noted that among all the edge detection algorithms, i.e. Local Threshold and Boolean Function Based Edge Detector, MarrHildreth Edge Detector, Sobel's Operator, Prewitt's Operator, Robert's Cross Operator and Canny Edge Detector, Canny edge detector provided with the most accurate results although it turned out to most time consuming. The proposed system has been tested and works well for up to four road crossings. Real time traffic light control using fuzzy logic based edge detector for images may further be expanded in future by inclusion of more number of test parameters, larger set of test cases and more number of roads from which live video feed can obtained simultaneously at any instant of time.

\section{ACKNOWLEDGMENTS}

With great pleasure we would like to express our profound gratitude and indebtedness to one and all at the School of Education Technology, Jadavpur University, Kolkata, for their continuous guidance, valuable advice and constant encouragement. Their valuable and constructive suggestions at many difficult situations are immensely acknowledged. We are in short of words to express their contribution to this paper through criticism, suggestions and discussions.

\section{REFERENCES}

[1] Pratt, W.K., Digital Image Processing, New York: Wiley-Interscience, 1978.

[2] Gonzalez, R.C., and Woods, R.E., Digital Image Processing, Addison- Wesley, Reading, M.A., 1992.

[3] Marr, D., and Hildreth, E.C., "Theory of edge detection", Proc. of the Royal Society of London, b207, 1980, 187217.

[4] Canny, J.F., "A computational approach to edge detection", IEEE Trans. on Pattern Analysis and Machine Intelligence, 8(6), 1986, 679-698.

[5] Smith, S.M., and Brady, J.M., "SUSAN - A new approach to low level image processing", International Journal of Computer Vision, 23(1), 1997.

[6] Hou, T. H. and Kuo, W.L., 1997. "A New Edge Detection method for Automatic Visual Inspection", International Journal of Advanced Manufacturing Technology, (1997) 13:407412 @ 1997 Springer-Verlag London Limited.

[7] Jiang, X. and Bunke, H. 1998. "Edge Detection in Range Images based on Scan Line Approximation", Computer Vision and Image Understanding ,Vol. 73, No. 2, February, pp. 183-199,1999 Article ID cviu.1998.0715, available online at http://www.idealibrary.com.

[8] Caragea S. 2008. Fondater, Administrator and Chief Editor, IntelliProject, "Difference Edge Detection", Licensed under IntelliProject open License, Romania, http://www.intelliproject.net.

[9] Genming, C. and Bouzong, Y., 1989. "A New Edge Detector with Thinning and Noise resisting Abilities", Journal of Electronics, China, Volume 6, No. 4, 314-319, DOI: $10.10071 \mathrm{BF} 02778914$.

[10] L. A. Zadeh, "Fuzzy sets," Information and Control, 8: 1965 , pp. 338-353.

[11] A. Kaufmann, "Introduction to the Theory of Fuzzy Subsets Fundamentals Theoretical Elements, Vol. 1. Academic Press, New York, 1975.

[12] L.C. Bezdek, "Pattern Recognition with fuzzy Objective Function Algorithm," Plenum Press, New York, 1981.

[13] K. Cheung and W. Chan, "Fuzzy One -Mean Algorithm for Edge Detection," IEEE Inter. Conf. On Fuzzy Systems, 1995, pp. 2039- 2044.

[14] Y. Kuo, C. Lee, and C. Liu, "A New Fuzzy Edge Detection Method for image Enhancement," IEEE Inter. Conf. on Fuzzy Systems, 1997, pp. 1069-1074.

[15] S. El-Khamy, N. El-Yamany, and M. Lotfy, "A Modified Fuzzy Sobel Edge Detector," Seventeenth National Radio Science Conference (NRSC'2000), February 2224, Minufia, Egypt, 2000. 
[16] M. Zhao, A. M. N. Fu, and H. Yan, "A Technique of Three-Level Thresholding Based on Probability Partition a Fuzzy 3-Partition". IEEE Trans. on Fuzzy Systems, vol.9, no.3, June 2001, pp. 469- 479.

[17] Bloch I., "Fuzzy sets in image processing", ACM Symposium on Allied Computing, 1994.

[18] Russo, F., and Ramponi, G., "Edge extraction by FIRE operators", Proc. of the 3rd IEEE Conference on Fuzzy Systems, 1994, 249-253.

[19] M. N. Mahani, M. K. Moqadam, H. N. pour, and A. Bahrololoom, "Dynamic Edge Detector Using Fuzzy Logic," CSISS' 2008, Sharif University of Technology, Kish, 2008, (In Persian).

[20] L. Liang and C. Looney, "Competitive Fuzzy Edge Detection," Applied Soft Computing, (3), 2003, pp. 123137.
[21] G. Mansoori and H. Eghbali, "Heuristic edge detection using fuzzy rule-based classifier," Journal of Intelligent and Fuzzy Systems, Volume 17, Number 5 / 2006, pp. 457- 469.

[22] Aijaz Ur Rahman Khan, Dr. Kavita Thakur, “ An Efficient Fuzzy Logic Based Edge Detection Algorithm for Gray Scale Image", International Journal of Emerging Technology and Advanced Engineering, Volume 2, Issue 8, August 2012, pp. 245-250

[23] Sreemana Datta, "Realtime Traffic Light Signalling Using Image Processing with Emergency Vehicle Detection and Traffic Signal Violation Detection", Scholars Journal of Engineering \& Technology, Volume $1(2)$, pp. 55-62.

[24] Sreemana Datta, "Comparative Study and Analysis of Various Edge Detection Algorithms in Digital Image Processing", Scholars Journal of Engineering \& Technology, Volume 1(2), pp. 78-90. 